\title{
Ectonucleotidases in Cancer and Inflammation
}

\author{
John Stagg, ${ }^{1}$ Linda F. Thompson, ${ }^{2}$ and Karen M. Dwyer ${ }^{3}$ \\ ${ }^{1}$ Centre de Recherche du Centre Hospitalier de l'Université de Montréal, Faculté de Pharmacie et Institut du Cancer de Montréal, \\ Montréal, QC, Canada H2L 4M1 \\ ${ }^{2}$ Immunobiology and Cancer Program, Oklahoma Medical Research Foundation, Oklahoma City, OK 73104, USA \\ ${ }^{3}$ Department of Medicine, Immunology Research Centre, The University of Melbourne, St Vincent's Hospital, \\ Melbourne, VIC 3065, Australia
}

Correspondence should be addressed to John Stagg, john.stagg@umontreal.ca

Received 2 October 2012; Accepted 2 October 2012

Copyright () 2012 John Stagg et al. This is an open access article distributed under the Creative Commons Attribution License, which permits unrestricted use, distribution, and reproduction in any medium, provided the original work is properly cited.

In 1970, Geoffrey Burnstock described the release of extracellular adenosine triphosphate (ATP) as a transmitter substance by nonadrenergic inhibitory nerves, and later in 1972, he formulated the hypothesis of purinergic neurotransmission [1]. Together with Che Su and John Bevan, Geoffrey Burnstock built the foundation to what was to become an entirely new field of research in biology [2]. While early studies focused on the role of purinergic receptors in neurotransmission, it soon became obvious that extracellular ATP and its hydrolyzed derivative adenosine had important roles in immune regulation. It is now recognized that purinergic signaling not only regulates neurotransmission and inflammation, but also influences diverse biological pathways, such as cell survival, proliferation, differentiation, lipid synthesis, and cell motility.

In 1978, Burnstock proposed two types of purinergic receptors: P1 receptors selective for adenosine and P2 receptors selective for ATP and ADP. In 1985, a pharmacological approach was proposed to distinguish between two types of $\mathrm{P} 2$ receptors: ionotropic $\mathrm{P} 2 \mathrm{X}$ and metabotropic $\mathrm{P} 2 \mathrm{Y}$ receptors [3]. Nucleotides, such as ATP, are released by a variety of cell types especially under stress conditions. Cancer cells, for instance, are known to release ATP in the tumor microenvironment. Nucleotides that are released in response to a stress signal can be hydrolyzed by membrane-bound enzymes called ectonucleotidases. By regulating the levels of extracellular nucleotides and nucleosides, ectonucleotidases are thus involved in numerous physiological and pathological conditions.

In this special issue, P. Chernogorova and R. Zeiser discuss the role of ectonucleotidases, in particular CD39

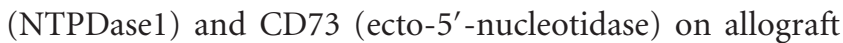
rejection, acute graft-versus-host disease (GvHD), and graftversus-leukemia (GvL) effect. Interestingly, recent evidence suggests that purinergic signaling influences the severity of alloimmune responses. As highlighted by P. Chernogorova and R. Zeiser, these studies suggest potential clinical use of recombinant ectonucleotidases or adenosine receptor agonists for regulation of alloimmune responses which can be tailored according to the clinical situation.

F. Ghiringhelli et al. review the current literature on the role of ectonucleotidases in tumor immune escape, with a focus on the immune regulatory function of Th17 cells. Th17 cells have emerged as key participants in a wide range of immune disorders and cancers. While the role of Th17 cells in cancer immunity remains controversial, F. Ghiringhelli et al. describe their recent seminal observation that ectonucleotidases are expressed on Th17 cells and that this is relevant in the context of tumor growth.

A. Battastini and colleagues present an overview of the various roles of purinergic signaling in gliomas. In gliomas, the presence of an inflammatory infiltrate is directly correlated with tumor malignancy, and this appears to be regulated in part by purinergic signaling. The authors discuss the observation that ATP triggers glioma cells to release proinflammatory factors important for the recruitment of monocytes and neutrophils, thereby favoring tumor growth. The authors discuss their own work that aims to establish whether ectonucleotidases are involved in macrophage polarization in gliomas.

A. Czopik and colleagues present a research article describing the protective role of CD73 in a model of colitis 
induced by administration of dextran sulfate sodium salt (DSS). The authors demonstrate that compared to wildtype mice, CD73-deficient gene-targeted mice are highly susceptible to DSS-induced colitis. The authors conclude that CD73 expression in the colon is critical for regulating the magnitude and the resolution of colonic immune responses.

K. M. Dwyer and colleagues discuss the role of the CD39adenosinergic axis in the pathogenesis of type 1 and type 2 diabetes. They discuss recent work suggesting that CD39 is involved in the pathophysiology of pancreatic dysfunction. This has importance clinical consequences, as drug development targeting different components of the pathway may be of relevance in the treatment of both type 1 and type 2 diabetes. The authors also discuss unanswered questions, such as what is the source of ecto- $5^{\prime}$-nucleotidase activity given the lack of CD73 expression within the pancreas.

J. Stagg and colleagues describe the role of CD73 and extracellular adenosine signaling in promoting tumor growth through paracrine and autocrine action. The authors discuss their recent work on the role of CD73 in spontaneous tumor growth and on the role of CD73 and adenosine on endothelial and immune cells.

In conclusion, this special issue on ectonucleotidases aims to acquaint investigators not familiar with this field with important recent advances with the hope of attracting scientists to join the effort of exploiting modulation of purinergic signaling for therapeutic purposes to treat cancer and inflammatory diseases.

John Stagg

Linda F. Thompson

Karen M. Dwyer

\section{References}

[1] G. Burnstock, "Purinergic nerves.," Pharmacological Reviews, vol. 24, no. 3, pp. 509-581, 1972.

[2] C. Su, J. A. Bevan, and G. Burnstock, " $\left[{ }^{3} \mathrm{H}\right]$ adenosine triphosphate: release during stimulation of enteric nerves," Science, vol. 173, no. 3994, pp. 336-338, 1971.

[3] M. P. Abbracchio, G. Burnstock, A. Verkhratsky, and H. Zimmermann, "Purinergic signalling in the nervous system: an overview," Trends in Neurosciences, vol. 32, no. 1, pp. 19-29, 2009. 

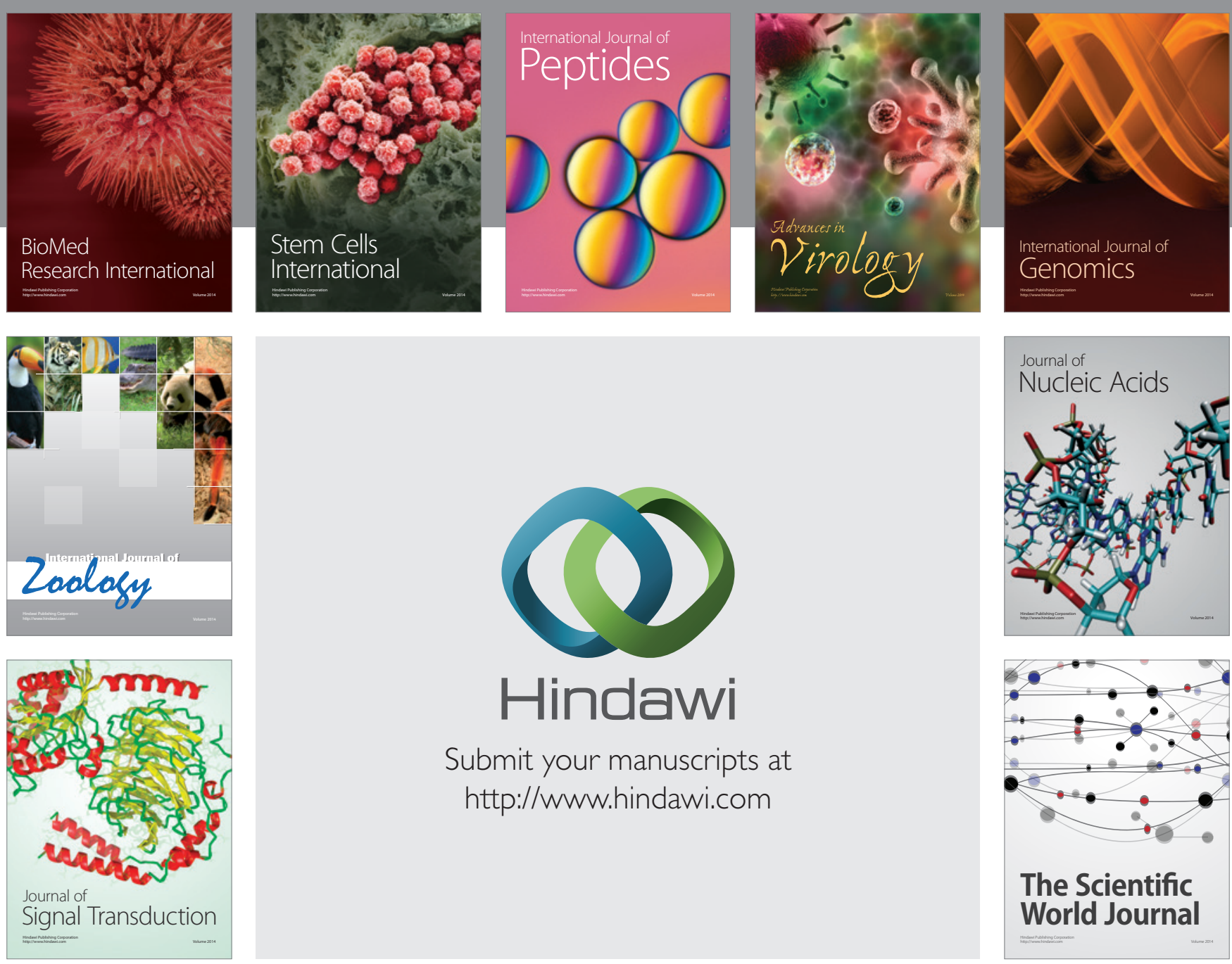

Submit your manuscripts at

http://www.hindawi.com
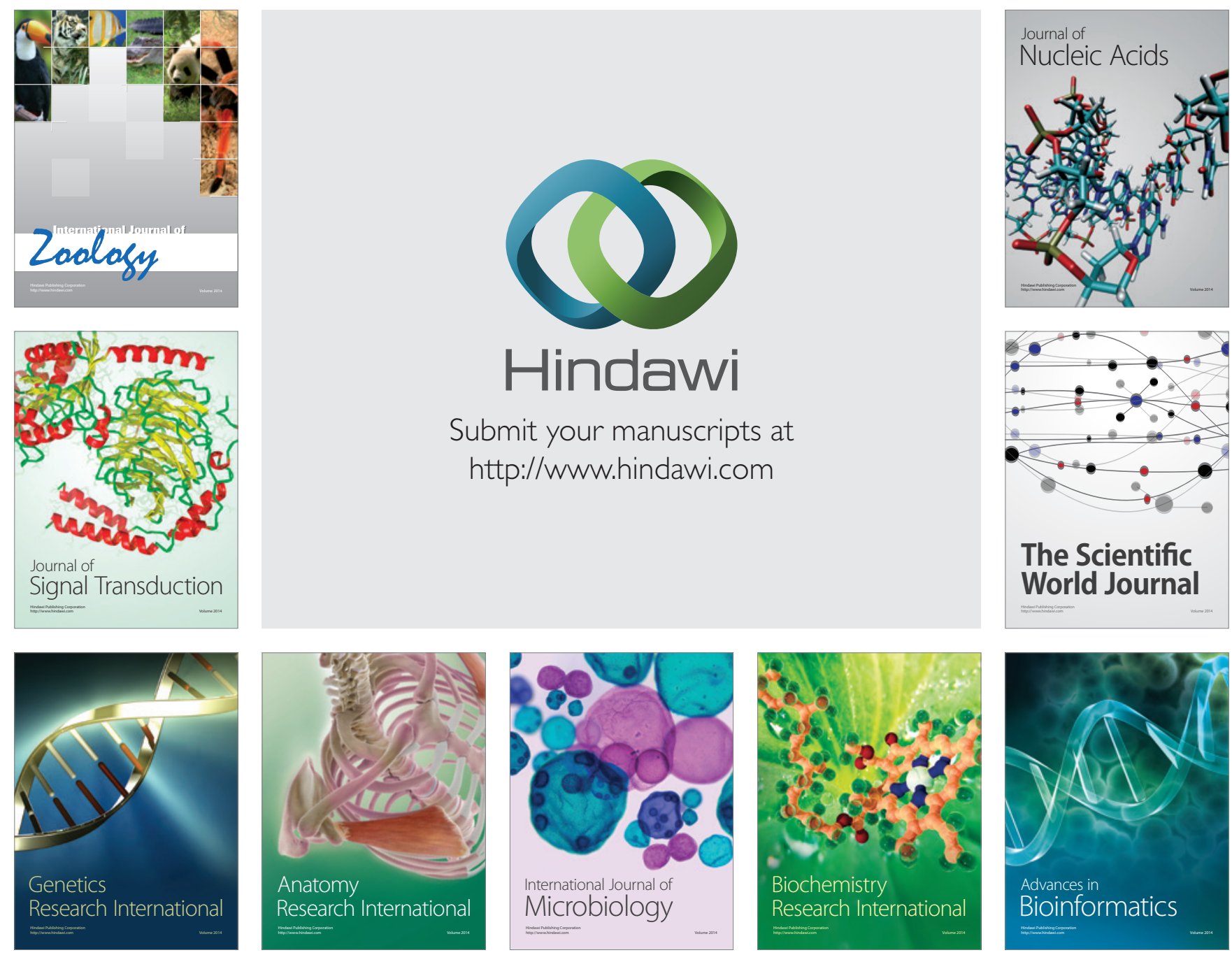

The Scientific World Journal
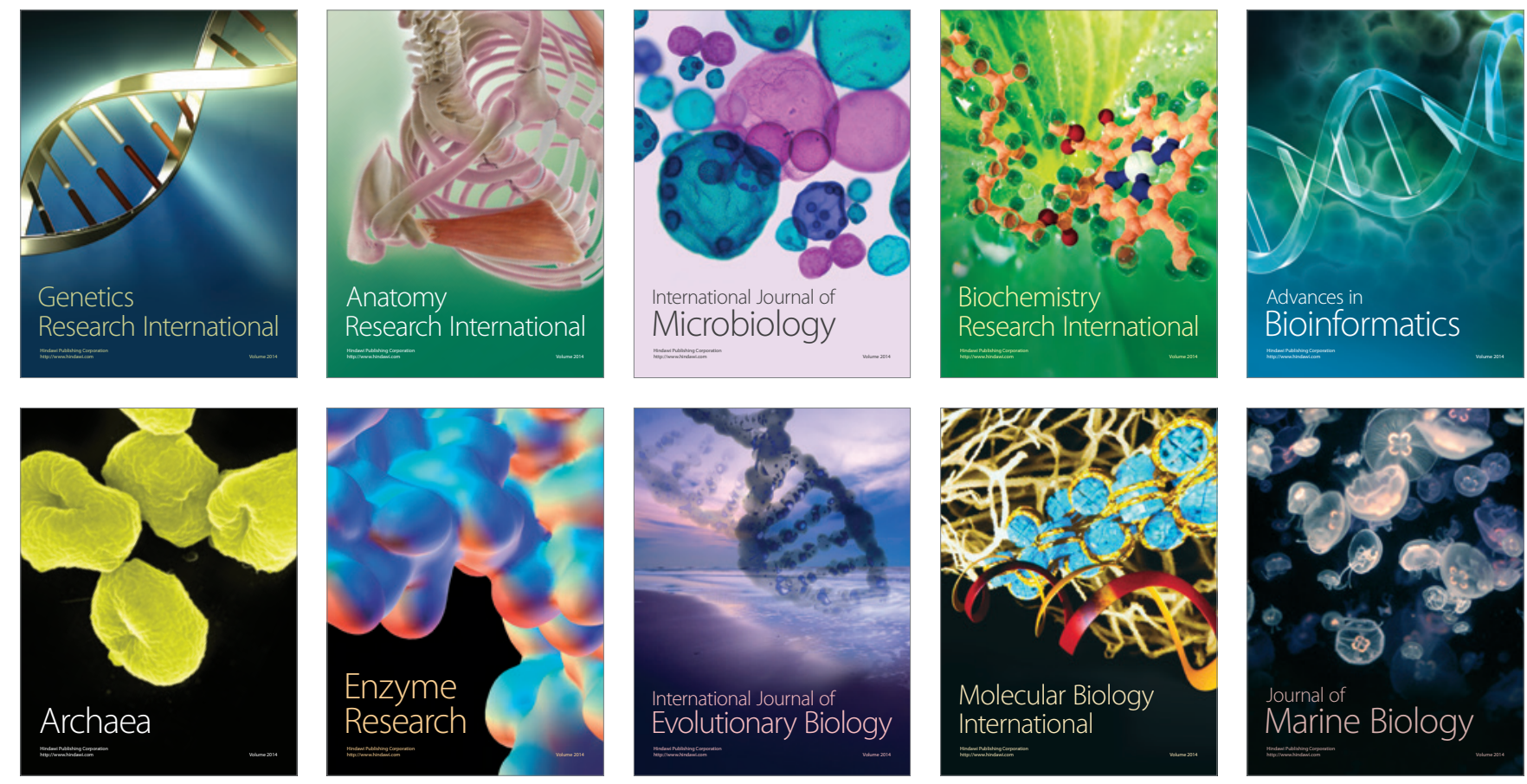